\title{
Taking Into Account Interactions between Converters in the Design of Aircraft Power Networks
}

\author{
Q.Li ${ }^{1}$, A.Formentini ${ }^{2}$, A.Baraston ${ }^{3}$, X. Zhang ${ }^{1}$, P.Zanchetta ${ }^{2}$, JL.Schanen ${ }^{3}$, D.Boroyevich ${ }^{1}$ \\ 1 Center for Power Electronics \\ Systems (CPES) \\ The Bradley Department of \\ Electrical \& Computer Engineering
}

Virginia Tech, Blacksburg, VA

24061, USA

\begin{abstract}
This paper presents some key interactions among converters, which need to be taken into account when designing a modern embedded electrical grid, including a large amount of Power Electronics based loads. A design by Optimization method is first used to define the converter parameters. During this step, it is mandatory to account for the interaction between the input and output EMI filters. The second step consists in designing the control strategy; the paper will show that the results are largely improved if all converters are considered simultaneously. Finally, the stability study of the embedded network has to be investigated. All these interactions are studied in the example of a three phases AC network, composed of a Voltage Source Inverter and an Active Front End.
\end{abstract}

Keywords-Power Electronics,Embedded Grids, EMC, Control, Stability, Design, Optimization,

\section{INTRODUCTION}

Designing a power grid does not simply mean connecting sources and loads to so-called power routes, insuring that the total power load demand can be satisfied by the sources. Indeed, modern power networks, in aircrafts for instance, are mainly composed of Power Electronics Converters, interfacing sources and loads to the grid [1], [2]. Therefore, power quality constraints (in the low frequency range), as well as EMC requirements (high frequency) impose adding specific filters. These filters interact with the converters regulations, leading to additional stability constraints. The easiest way to design the power grid should be increasing the filters, in order to prevent any interactions between converters through the grid; this way, all converters could be sized independently. Unfortunately, this is not compatible with the weight reduction demand of aircraft manufacturers. The process becomes thus quite challenging and interactions studies at network level are mandatory. On a basic example of two connected converters, this paper will introduce and discuss several interactions on an AC network and suggest design methodologies.

The proposed test bench topology is a basic AC networks, illustrating some possible interactions between converters. A Voltage Source Inverter (VSI) generates an AC bus, used to conveying electrical power and is connected to one specific load: an Active Front End (AFE) converter, generating a DC bus, used for any other dc load. As displayed in Fig. 1-Top, all filters and passive elements are to be sized, as well as control loops.

To simplify the study, the VSI will be considered fixed (all passive elements and control system parameters). The study will start with the design of the other converter, which is the AFE. For this purpose, a Design by Optimization methodology will be considered [2]. Section II will briefly introduce this methodology, and especially point out the interactions between the input and output EMI filters (Electromagnetic Interference), which prevents the use of conventional design methods. Once the converter has been designed (i.e. all passive elements are sized to reach the minimum weight), the control design step will be introduced in section III. Interactions between the VSI and the AFE will be underlined. Section IV will present the final validation of the embedded grid design, which is the stability study. In the conclusion, a prospective Design by Optimization method at grid level will be presented.

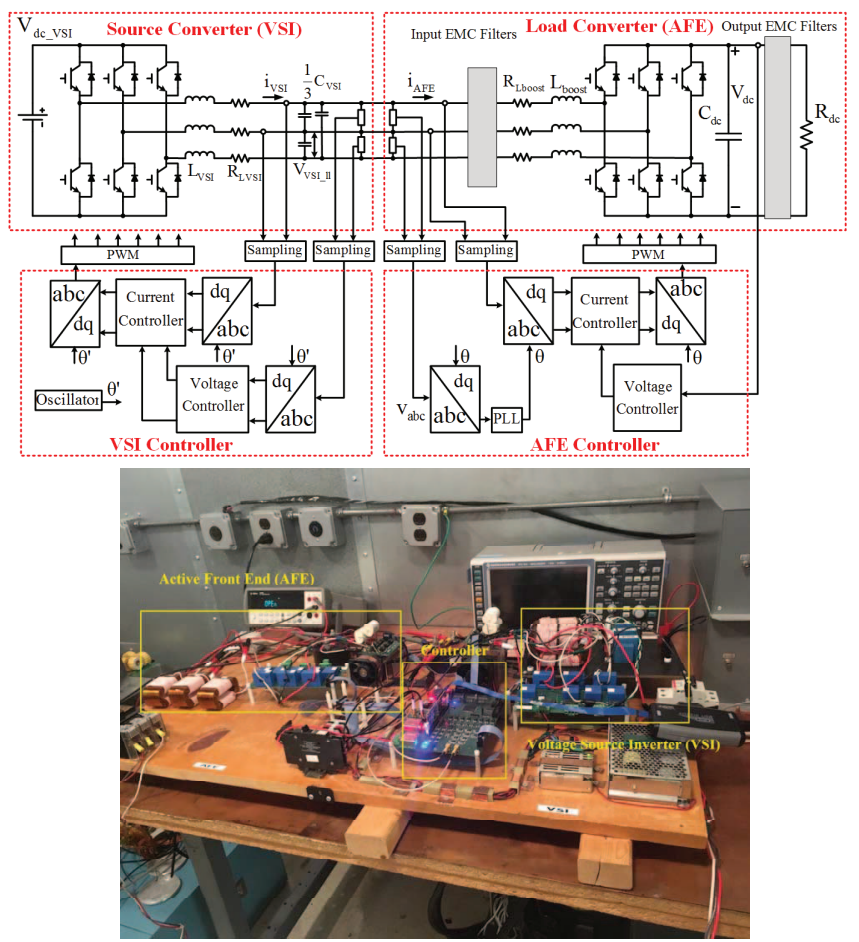

Fig. 1. AC grid used for illustration, composed of a Voltage Source Inverter (VSI) and an Active Front End (AFE) [3]. 


\section{Design by Optimization Applied to the AFE}

In this application, the AFE parameters to be designed are the three phases boost inductors $\mathrm{L}_{\text {boost, }}$ the output DC capacitor C_DC, the switching frequency and of course the EMC filters on both $\mathrm{AC}$ and DC sides. In a first step, the switching frequency has been imposed at $20 \mathrm{kHz}$. The design criterion for the boost inductors and the DC capacitor have not been considered with the usual current and voltage ripple (e.g 5\% or $10 \%$ ), but through the effect of these ripples on EMC and losses. The input current ripple increases the semiconductor losses (due to increased peak current), which are limited in the design process. Furthermore, both $\mathrm{L}_{\text {boost }}$ and $\mathrm{C}$ DC contribute to EMC disturbances path: their values will thus be considered during the EMC analysis. Finally, several internal constraints, such as maximum losses and non-saturation of the magnetic material for the inductor, or maximum RMS current for the capacitor, need also to be taken into account during the design of Lboost and C DC, using the same ideas as in [3]. The EMC filters to be determined are described in Fig. 2, with respect to existing standards as the DO160 in aircraft applications, for both AC and DC networks [5]. The EMC design is performed considering that the AFE is connected to a Line Impedance Stabilization Network (LISN) [6] on both AC and DC sides. Like the previous passive elements, the design of these filter elements (Cx_AC, Cy_AC, LDM_AC, LCM_AC, Cx_DC, Cy DC, LDM DC, LCM DC) has also to account for technological constraints, as limitations due to losses, saturation, etc.

Generic design models using analytical formulas have been developed for capacitors and inductors/transformers [7]. The capacitor model is an interpolation of manufacturer datasheets, providing all necessary design parameters, its parasitic elements (e.g. the Equivalent Series Inductance Esl) and the weight as a function of the capacitance. For the magnetic components, the design parameters are based on a technological description: the toroidal core geometry and the turn number. The magnetic material permeability can also be chosen.

To evaluate the EMC behaviour of the converter, it is not possible to use the conventional method, considering the bare noise and defining the necessary attenuation to be provided by the EMI filter. Indeed, both input and output filters interact in Common Mode [8] [9]. As an illustration, Fig 3 shows how the EMC spectrum on the AC side is modified, when considering or not the EMI filter on the DC side. Therefore, the design of the input filter depends on the output one, and vice versa.

Consequently, it is mandatory to solve the EMC issues from a global point of view, considering simultaneously both $\mathrm{AC}$ and DC filters. For this purpose, the equivalent sources method has been used [10]. It replaces all the switching devices by equivalent current and voltage sources, reproducing their discontinuities (Fig. 4). The circuit can thus be solved in the frequency domain; this leads to quick computation times and derivability of the model, which is suitable to be used in an optimization process.

Once all equations have been determined to express all the constraints (passives, EMC filters), an optimization algorithm determines the geometrical parameters of all inductors and all capacitor in order to provide the minimum weight. The simplified illustration of the AFE optimization method is provided in Fig. 5. Several functions have been developed and are not illustrated here, such as getting the commutation waveforms, computing RMS values in the capacitors, designing the inductors, etc. All models have been imposed to be derivable in order to be able to use gradient based optimization algorithms, as detailed in [7]. The optimization environment is CADES, allowing simple and automatic derivation of the models [11], with respect to optimization variables, but also all constraints.

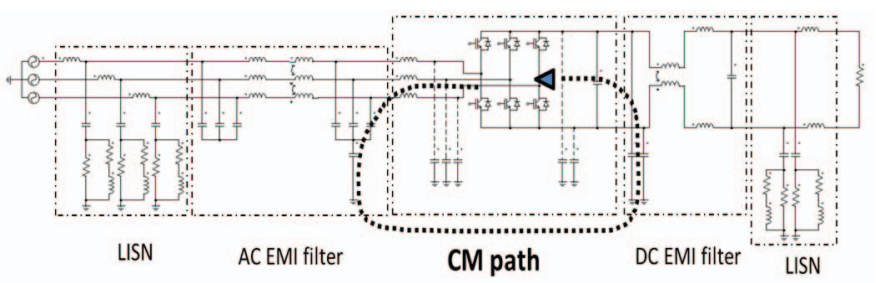

Fig. 2. Illustration of the Common Mode interaction between $\mathrm{AC}$ and DC EMI filters.

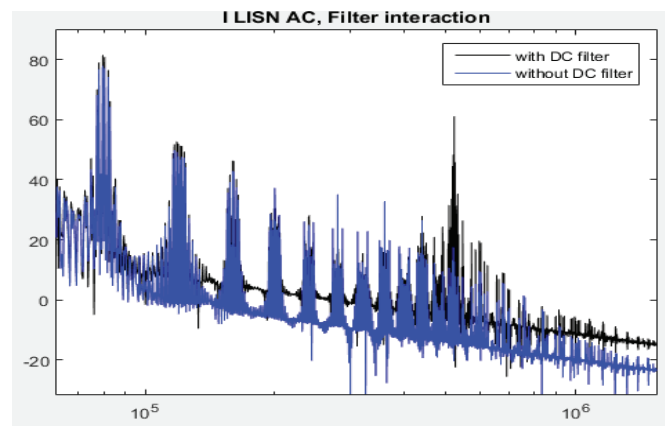

Fig. 3. Comparison of the EMC spectrum measured on one branch of the LISN on the AC side, with and without the DC EMI filter.

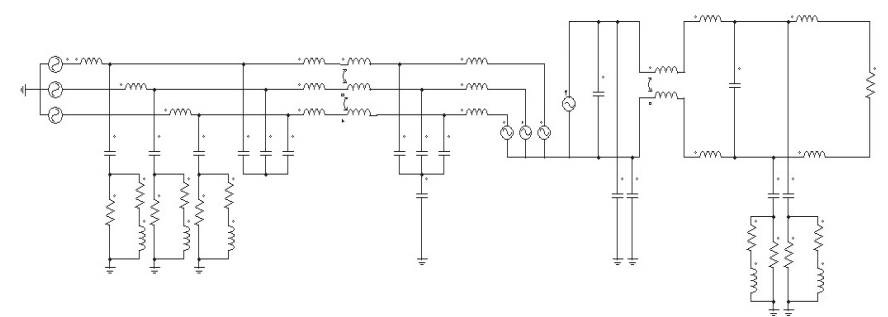

Fig. 4. Equivalent EMC circuit of the AFE with equivalent disturbance sources, EMI filters and LISN. All stray elements are not displayed for clarity.

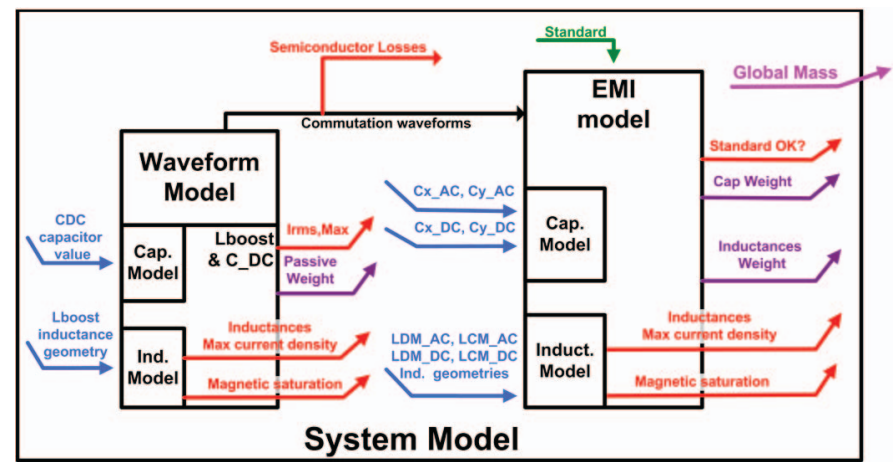

Fig. 5. Illustration of the Design by Optimization method used for the AFE 
The optimized values of the converter parameters are given in TABLE I. The values used for the inverter (fixed) are provided in TABLE II. Besides these optimization results, some specific optimization tests have been carried out with fixed values of boost inductor $\mathrm{L}_{\text {boost, }}$ in order to check the impact of either low or high values on the control and stability aspects of the grid.

TABLE I. AFE OPTIMIZATION RESULTS

\begin{tabular}{|c|c|c|}
\hline \multicolumn{3}{|c|}{ Fixed parameters } \\
\hline Power & \multicolumn{2}{|l|}{$760 \mathrm{~W}$} \\
\hline Input/Output Voltage & \multicolumn{2}{|c|}{$99.6 \mathrm{~V}$ line-line $400 \mathrm{~Hz} / 270 \mathrm{~V} \mathrm{DC}$} \\
\hline Switching Frequency & \multicolumn{2}{|l|}{$20 \mathrm{kHz}$} \\
\hline \multicolumn{3}{|c|}{ Optimized parameters } \\
\hline Name & Value & Weight \\
\hline C_DC & $4.5 \mu \mathrm{F}$ (RMS current: $3 \mathrm{~A}$ ) & $5.9 \mathrm{~g}$ \\
\hline $\begin{array}{ll}\text { Lboost }(* 3) \\
-\quad & \text { Core Height } H \\
- & \text { Internal Diameter ID } \\
- & \text { External Diameter OD } \\
- & \text { Turn Number } N \\
- & \text { Permeability } \mu \\
\end{array}$ & $\begin{aligned} 225 \mu \mathrm{H} & \\
- & 4 \mathrm{~mm} \\
- & 12.3 \mathrm{~mm} \\
- & 20.8 \mathrm{~mm} \\
- & 73 \\
- & 102\end{aligned}$ & $\begin{array}{l}11 \mathrm{~g} \\
(* 3)\end{array}$ \\
\hline $\begin{array}{l}\text { AC EMI filter } \\
\text { Cx_AC } \\
\text { Cy_AC } \\
\text { LCM_AC } \\
\text { (3 phases coupled) } \\
\\
\text { LDM_AC }\end{array}$ & $\begin{array}{l}251 \mathrm{nF} \\
7.6 \mathrm{nF} \\
3.9 \mathrm{mH} \\
H=6.75 \mathrm{~mm}, I D=27.0 \mathrm{~mm}, \\
O D=33.7 \mathrm{~mm}, \text { Nphase }=34, \\
\mu=20000 \\
\text { LCM_AC leakage } 53.5 \mu \mathrm{H}\end{array}$ & $\begin{array}{l}3 \mathrm{~g}(* 3) \\
\approx 0 \\
27 \mathrm{~g}\end{array}$ \\
\hline $\begin{array}{l}\text { DC EMI filter } \\
\text { CX_DC } \\
\text { Cy_DC } \\
\text { LCM_DC } \\
\text { (Plus-Minus coupled) }\end{array}$ & $\begin{array}{l}362 \mathrm{nF} \\
10 \mathrm{nF} \\
1.48 \mathrm{mH} \\
H=7.06 \mathrm{~mm}, I D=28.2 \mathrm{~mm}, \\
O D=35.3 \mathrm{~mm}, \text { Nphase }=16, \\
\mu=34465 \\
\text { LCM_DC leakage } 26.1 \mu \mathrm{H}\end{array}$ & $\begin{array}{l}4 \mathrm{~g}(* 2) \\
\approx 0 \\
21 \mathrm{~g}\end{array}$ \\
\hline
\end{tabular}

TABLE II. VSI FIXED VALUES

\begin{tabular}{|l|c|}
\hline \multicolumn{2}{|c|}{ Fixed parameters } \\
\hline Power & $760 \mathrm{~W}$ \\
\hline Input/Output Voltage & $270 \mathrm{~V} \mathrm{DC} / 99.6 \mathrm{~V}$ line-line $400 \mathrm{~Hz}$ \\
\hline Switching Frequency & $20 \mathrm{kHz}$ \\
\hline Output Inductance of VSI L $\mathrm{VSI}$ & $970 \mu \mathrm{H}(120 \mathrm{~m} \Omega$ series resistance $)$ \\
\hline Output Capacitance of VSI C & $31.8 \mu \mathrm{F}$ \\
\hline
\end{tabular}

\section{CONTROL DESIGN}

One of the most common control design procedures for interconnected AC system is to design the controllers of every single network components separately and then use large enough passive components to minimize the interaction between the network subsystems.
This approach however is not suitable for aerospace applications where the weight minimization is one of the main goals in the network design process. For this reason a design procedure that keeps in consideration the whole network should be adopted. Since the goal of this paper is to show the network interactions on the AC side, in the proposed design procedure the simplified system of Fig. 6 has been modelled in the dq reference frame neglecting the AFE DC side voltage control loop. The resulting system equation are reported in the following

$$
\dot{\tilde{x}}=\tilde{A} \tilde{x}+\tilde{B} \tilde{u}
$$

where

$$
\begin{aligned}
& \tilde{A}=\left[\begin{array}{cccccc}
-\frac{R_{i}}{L_{i}} & -\frac{1}{L_{i}} & \omega & 0 & 0 & 0 \\
\frac{1}{C_{i}} & 0 & 0 & \omega & \frac{1}{C_{i}} & 0 \\
-\omega & 0 & -\frac{R_{i}}{L_{i}} & -\frac{1}{L_{i}} & 0 & 0 \\
0 & -\omega & \frac{1}{C_{i}} & 0 & 0 & \frac{1}{C_{i}} \\
0 & -\frac{1}{L_{a}} & 0 & 0 & -\frac{R_{a}}{L_{a}} & \omega \\
0 & 0 & 0 & -\frac{1}{L_{a}} & -\omega & -\frac{R_{a}}{L_{a}}
\end{array}\right] \\
& \tilde{B}=\left[\begin{array}{cccc}
\frac{1}{L_{i}} & 0 & 0 & 0 \\
0 & 0 & 0 & 0 \\
0 & \frac{1}{L_{i}} & 0 & 0 \\
0 & 0 & 0 & 0 \\
0 & 0 & \frac{1}{L_{a}} & 0 \\
0 & 0 & 0 & \frac{1}{L_{a}}
\end{array}\right] \\
& \tilde{x}=\left[\begin{array}{llllll}
I_{i d} & V_{c d} & I_{i q} & V_{c q} & I_{a d} & I_{a q}
\end{array}\right]^{T} \\
& \tilde{u}=\left[\begin{array}{llll}
V_{i d} & V_{a d} & V_{i q} & V_{a q}
\end{array}\right]^{T}
\end{aligned}
$$

$R_{a}$ and $L_{a}$ ( $\left.\mathrm{L}_{\text {boost }}+\mathrm{L}_{-} \mathrm{DM} \_\mathrm{AC}\right)$ are the resistance and inductance of the AFE input filter while $\omega$ is the system frequency in abc domain. $I_{i}$ and $V_{c}$ are the VSI filter inductor current and capacitor voltage while $I_{a}$ is the AFE filter inductor current. $V_{i}$ and $V_{a}$ are the voltages generated by the VSI and AFE respectively. The subscript $d$ and $q$ denote quantity related to the d-axis and q-axis respectively.

Since in this work the VSI side has been considered fixed, its controller has been designed as a standalone system and kept constant for the whole optimization process. A dq reference frame PI-based cascade control structure with an inner current loop and an outer voltage loop has been used. The system (1) has been then rewritten including the VSI controller and the integral states of the AFE controller resulting in equation (3) hereafter: 


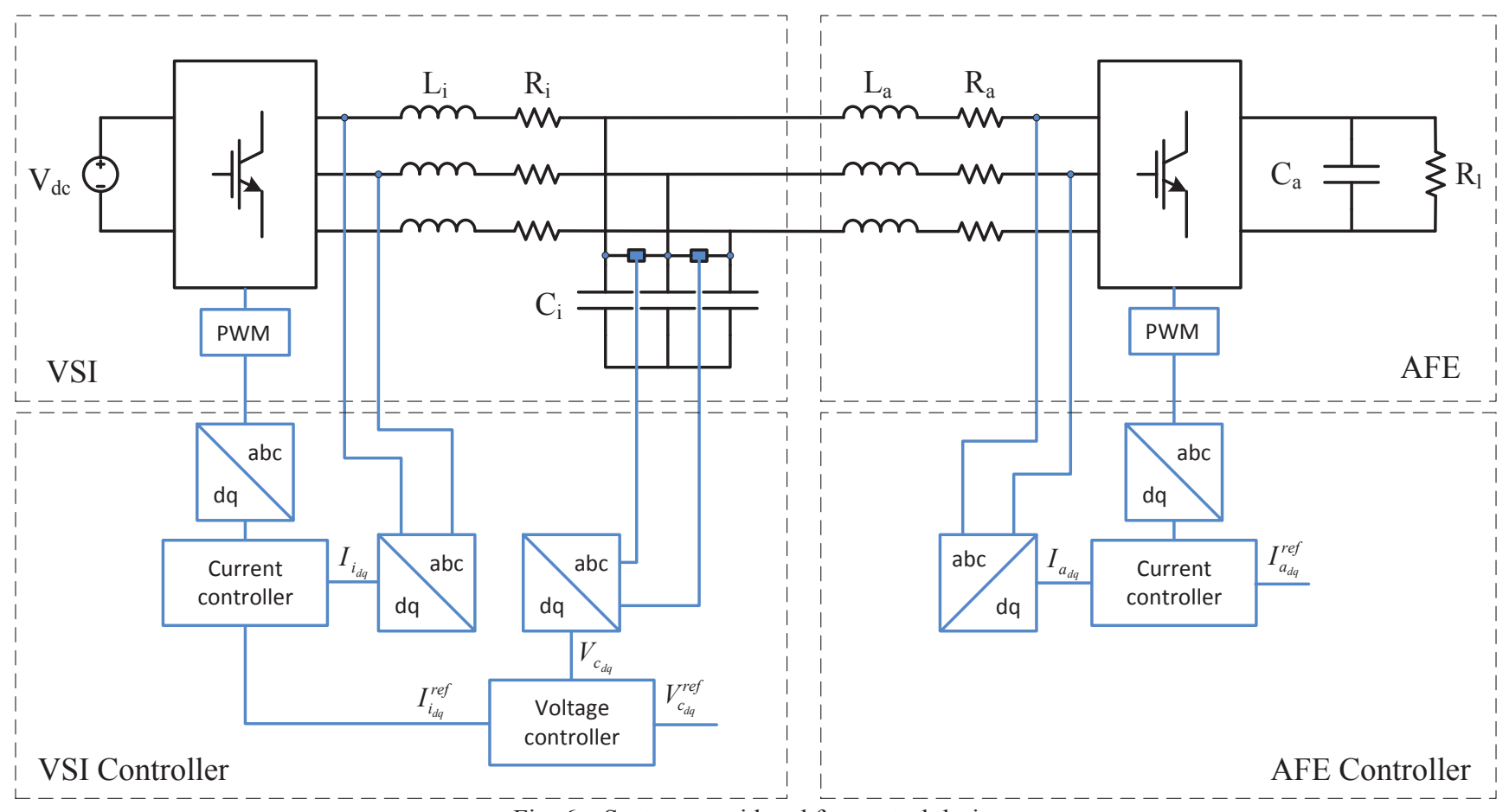

Fig. 6 - System considered for control design

$$
\dot{x}=A x+B u
$$

where

$$
\begin{aligned}
& x=\left[\begin{array}{ll}
x_{v s i} & x_{a f e}
\end{array}\right]^{T} \quad u=\left[\begin{array}{ll}
V_{a d} & V_{a q}
\end{array}\right]^{T} \\
& x_{v s i}=\left[\begin{array}{llllllll}
I_{i d} & V_{c d} & I_{i q} & V_{c q} & W_{v d} & W_{i d} & W_{v q} & W_{i q}
\end{array}\right]^{T} \\
& x_{a f e}=\left[\begin{array}{llll}
I_{a d} & I_{a q} & W_{a d} & W_{a q}
\end{array}\right]^{T}
\end{aligned}
$$

$W_{v}$ and $W_{i}$ are VSI-side voltage and current loop integral states respectively, while $W_{a}$ are the integral states on the AFE side. $A \in \mathbb{R}^{12 \times 12}$ and $B \in \mathbb{R}^{12 \times 2}$ are the complete system state and control matrices respectively. To design a controller for the AFE side requires solving a structured state feedback problem [12]. Namely, it consists in finding the optimal state feedback gain $K$ that minimizes:

$$
J(K)=\int_{0}^{\infty} x^{T} Q x+u^{T} R u d t, \quad u=K x_{a f e}
$$

$\mathrm{Q}$ and $\mathrm{R}$ represent the state and control weighting matrices respectively. During the optimization process the structure of the feedback gain matrix it is forced to be

$$
K \in\left[\begin{array}{cccc}
K_{p} & 0 & K_{i} & 0 \\
0 & K_{p} & 0 & K_{i}
\end{array}\right]
$$

where $K_{p}$ and $K_{i}$ are the arbitrary proportional and integral gains to be tuned during the optimization process.
Fig. 7 shows the transient response of the system to a connection of a resistive load to the AC grid (in parallel to the AFE) with three different values of the AFE filter inductor, chosen to cover a wide range of possible values in the space of solutions, the second corresponding to the design in TABLE I. . Figures report the system response with two different tuning methods. Yellow and purple tracks are Id and Iq currents when AFE current controller is tuned with the global method proposed above. Blue and red tracks are Id and Iq when AFE current controller is tuned with a standard LQR method neglecting VSI dynamics and considering the AFE converter connected to an ideal grid. The same values of $\mathrm{Q}$ and $\mathrm{R}$ matrices have been used in the two tuning processes. It is possible to notice that the proposed approach returns a faster and better damped response independently from the inductor boost value compared with a standalone tuning procedure. Not surprisingly, the increase of the boost inductance moderates the difference between the global tuning vs the independent one, since interactions between converters are reduced.

Table III: AFE current controller parameters

\begin{tabular}{|c|cc|cc|}
\hline & \multicolumn{2}{|c|}{ Global tuning } & \multicolumn{2}{c|}{ Local tuning } \\
\hline Controller gains & $\mathrm{Kp}$ & $\mathrm{Ki}$ & $\mathrm{Kp}$ & $\mathrm{Ki}$ \\
\hline AFE La 1032 $\boldsymbol{\mu H}$ & 15.398 & 7670 & 7.2634 & 5725 \\
\hline AFE La 278.5 $\boldsymbol{\mu H}$ & 14.02 & 7562 & 3.346 & 4745 \\
\hline AFE La 57 $\boldsymbol{\mu H}$ & 16.715 & 8764 & 0.6116 & 3474 \\
\hline
\end{tabular}

Table IV: VSI and AFE dc-link controller parameters

\begin{tabular}{|c|c|c|}
\hline Controller & $\mathbf{K p}$ & $\mathbf{K i}$ \\
\hline VSI Current & 12 & $3.83 \mathrm{e} 4$ \\
\hline VSI Voltage & 0.0799 & 50 \\
\hline AFE DC Voltage & 0.16755 & 52.63 \\
\hline
\end{tabular}



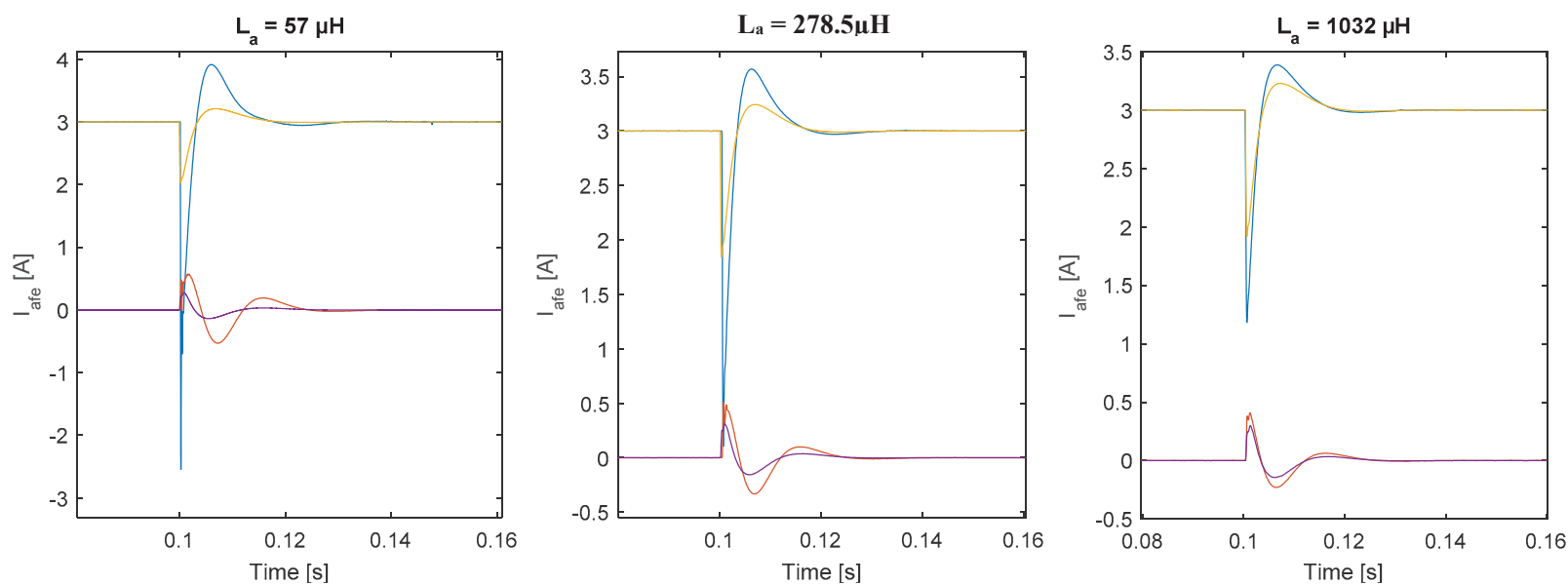

Fig. 7: System response to a resistive load connection on the AC grid with three different AFE inductor values $(57 \mu \mathrm{H}, 278.5 \mu \mathrm{H}$ and $1032 \mu \mathrm{H})$. Blue and red tracks are $\mathrm{I}_{d}$ and $\mathrm{I}_{q}$ when the AFE current controller is tuned standalone. Yellow and purple tracks are $\mathrm{I}_{d}$ and $\mathrm{I}_{q}$ when the AFE current controller is globally tuned.

\section{STABILITY StUdy}

System stability is one of the most important considerations when designing an electrical system. After the optimization design process of the EMC filters and the globaloptimized selection of the control parameters, stability of the VSI-AFE electrical system is analyzed using the generalized Nyquist stability criterion (GNC) [13], [3]. GNC is now a commonly-used method for stability analysis at the $\mathrm{AC}$ interface, by studying the return-ratio matrix of source converter output-impedance and load converter inputadmittance [13].

An equivalent circuit of the VSI-AFE converter system is shown in Fig. 8 to illustrate the principle of applying GNC for system stability study. In this paper, stability analysis is conducted in $\mathrm{d}-\mathrm{q}$ synchronous reference frame. From the figure, the interface voltage matrix $\mathbf{V}_{\mathbf{i}}$ is expressed as [13]:

$$
V_{i}(\mathrm{~s})=\left(I+Z_{s d q}(s) \cdot Y_{L d q}(s)\right)^{-1} V_{s d q}(s) .
$$

System stability can then be evaluated by studying the characteristic functions (eigenvalues) of the return-ratio matrix L(s):

$$
\mathbf{L}(\mathrm{s})=Z_{s d q}(s) \cdot Y_{L d q}(s) .
$$

$\mathbf{L}(\mathbf{s})$ is a two-by-two matrix in this specific case study. Thus, two characteristic functions $\left[l_{1}(s) ; l_{2}(s)\right]$ are associated with $\mathbf{L}(\mathbf{s})$ and the characteristic loci of these eigenvalues will be used in the system stability analysis.

To calculate the output-impedance of the voltage source inverter as well as the input-admittance of the active front end, small-signal models of the converters are firstly derived in $d-q$ frame. As the voltage source inverter is considered fixed in the previous sections (with predesigned output filters and known control parameters), its output-impedance remains unchanged and the corresponding bode plot is shown in Fig. 9. For the active front end, EMC filters are added to both the ac and dc sides of the converter to reduce the transfer of electromagnetic noise between the converters and the load. Fig. 11 presents the small-signal model of the boost rectifier with EMC filters and controllers. Only differential mode filters have been considered, CM effects being neglected in this stability study. For numerical applications, $\mathrm{L}_{\text {boost }}$ and L_DM_AC has been chosen according to TABLE I. . Based on the information above, the following discussion will focus on studying the stability of the designed VSI-AFE system and analyzing the effects of EMC filters on the load-converter input-admittance.

Without EMC filters, the open-loop small-signal model of the AFE is described by a set of three-dimensional state-space equations. The three states, namely $\tilde{l}_{d 1}, \tilde{l}_{q 1}$, and $\tilde{v}_{d c}$, are introduced by the boost-inductors and the output DC capacitor. The input EMC filters, however, add four additional

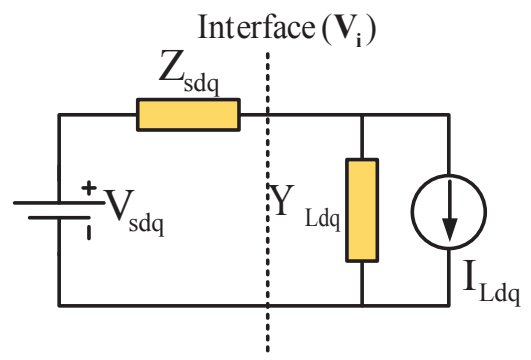

Fig. 8. An equivalent circuit of VSI-AFE converter system in d-q frame.

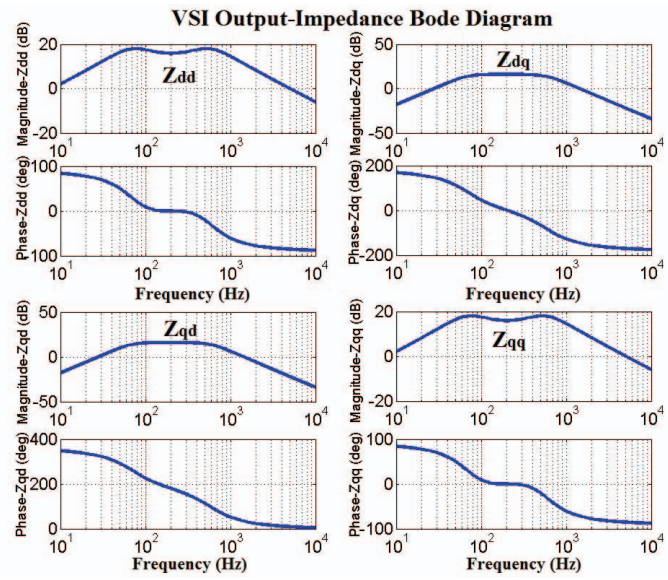

Fig. 9. Bode diagram of the VSI output impedance (close-loop). 


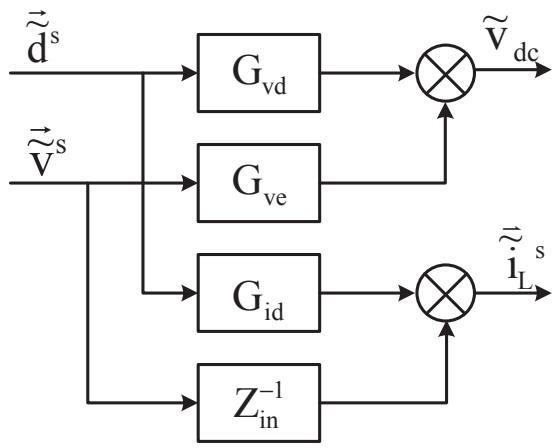

Fig. 10. Open-loop transfer function flow chart of AFE.

states to the small-signal model, which influence the AFE input-admittance. The open-loop transfer function block scheme of the active front end is shown in Fig. 10. By adopting the input EMC filters, the transfer functions of the open-loop system $G_{v d}$ (from $\tilde{\vec{d}}$ to $\tilde{v}_{d c}$ ), $G_{v e}$ (from $\tilde{\vec{v}}^{s}$ to $\tilde{v}_{d c}$ ), $G_{i d}$ (from $\tilde{\vec{d}}$ to $\tilde{\vec{\imath}}_{L}$ ) and $Z_{\text {in }}^{-1}$ (from $\tilde{\vec{v}}^{s}$ to $\tilde{\vec{\imath}}_{L}$ ) need to be recalculated. Bode plots of the close-loop AFE inputadmittance with and without the input EMC filters are drawn together in Fig. 12. By comparison, it is concluded that the input EMC filters will influence the high-frequency performance of the AFE input-impedance. Influence of the output EMC filters is analyzed following a similar process and it is observed that the output EMC filters have a minor effect on the system stability.
After calculating the VSI output-impedance and the AFE input-admittance, the stability of the targeted system is analyzed using GNC. As both the $\boldsymbol{Z}_{\boldsymbol{s} \boldsymbol{d q}}(\boldsymbol{s})$ and the $\boldsymbol{Y}_{\boldsymbol{L d q}}(\boldsymbol{s})$ are diagonal-dominated two-by-two matrices, a simplified GNC is implemented instead in the stability analysis process [13]. In this case, the return-ratio matrix $\mathbf{L}(\mathbf{s})$ is transformed into:

$$
\mathbf{L}(\mathbf{s})=\left[\begin{array}{cc}
Z_{d d_{-} V S I} \cdot Y_{d d_{-} A F E} & 0 \\
0 & Z_{q q_{-} V S I} Y_{q q_{-} A F E}
\end{array}\right]
$$

and the corresponding characteristic functions are derived as:

$$
l_{1}(s)=Z_{d d_{-} V S I} \cdot Y_{d d_{-} A F E} ; \quad l_{2}(s)=Z_{q q_{-} V S I} \cdot Y_{q q_{-} A F E} .
$$

Finally, the characteristic loci of $\mathbf{L}(\mathbf{s})$ are plotted in Fig. 13 for both cases (with and without input EMC filters). By applying GNC to the Nyquist plot, it is proved that the designed VSIAFE system is stable. To further verify this result, converter system simulation was carried out and the results are presented in Fig. 14. Fig. 14 (a) shows the waveforms of the AC output voltage from the voltage source inverter; the DC output voltage from the active front end is drawn in Fig. 14 (b). The control system of the AFE starts to function at 0.1 second. After a short settling period, the entire system goes to a stable state, which demonstrates the validity of the GNC stability criterion.

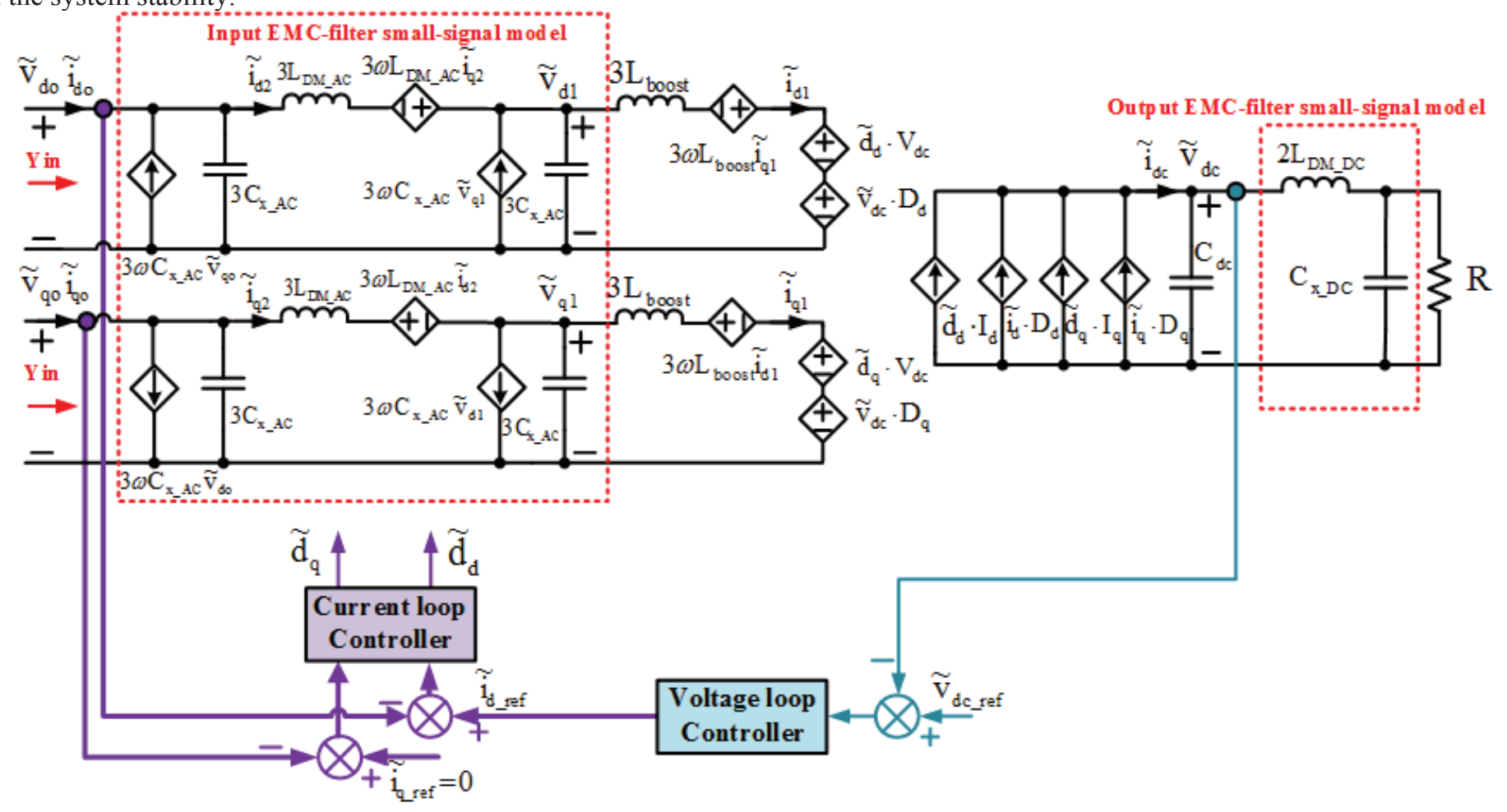

Fig. 11. Small-signal model of AFE with controllers. 

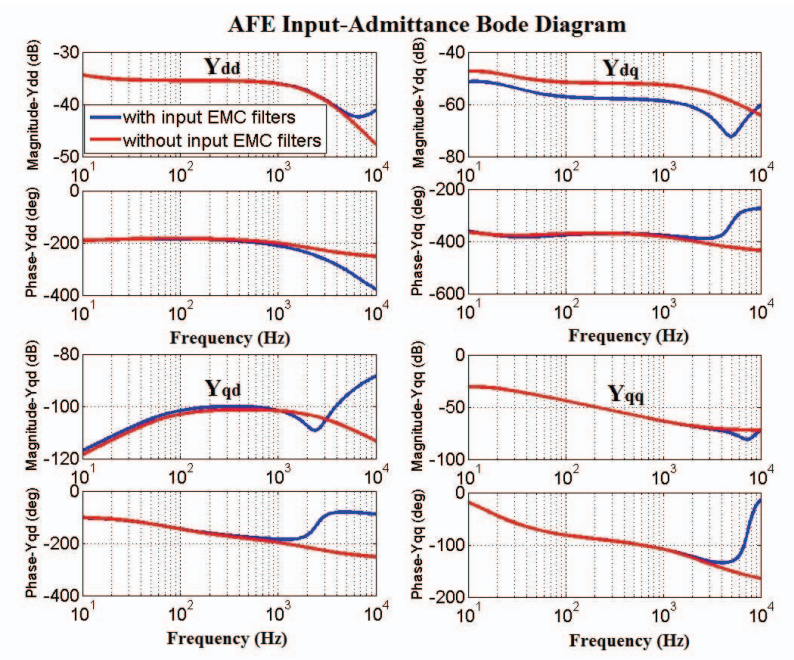

Fig. 12. Bode diagram of the AFE input admittance (close-loop).

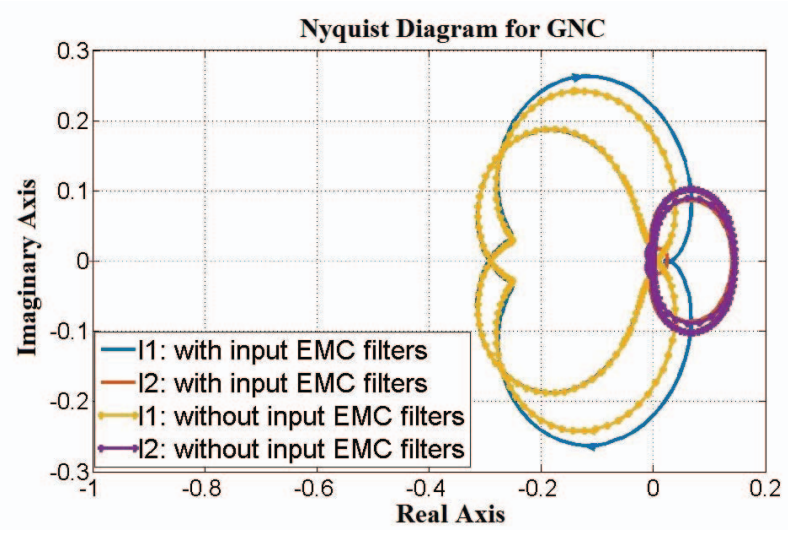

Fig. 13. Nyquist diagram of the converter system for GNC analysis.
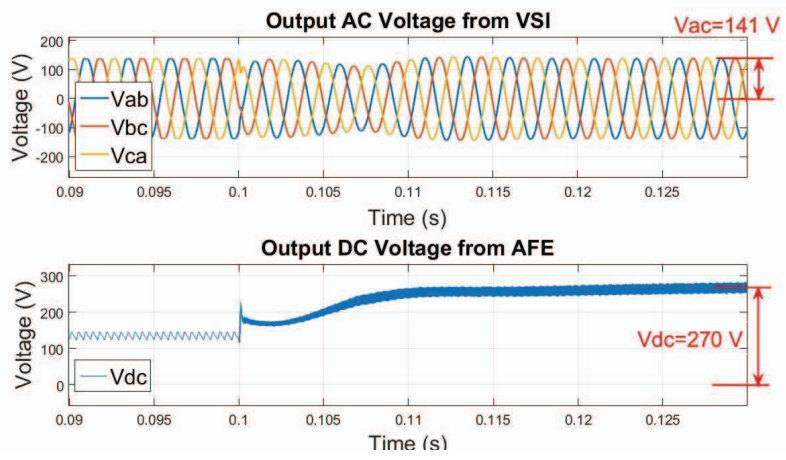

Fig.14. Simulation results of the VSI-AFE system: (a) output AC voltage from VSI; b) output DC voltage from AFE.

\section{CONCLUSION}

This paper has studied several aspects to be investigated when designing a modern embedded network, involving power electronics loads. The first point is to design the converters. For this purpose, a design by optimization method seems promising, taking into account all interactions at converter level. In this paper, specific attention has been paid to the interaction between input and output EMI filters, that prevents using conventional sizing methods, supposing that theses filters can be sized independently. Once the converters have been designed, their regulation has to be tuned in order to respect the dynamic requirements. This paper has shown that an independent control design for each converter does not lead to the best performances, since the passive elements linking all converters are not infinite, thus interactions occurs between the local systems. A global approach is thus a better choice. Finally, with all converters and control being defined, the stability of the network has to be guaranteed. For this purpose, the GNC criterion has been used, taking into account all elements of the network, including the EMC filters. Even if the difference is small in this application, this effect is important during optimization procedures, since it may help in rejecting some solutions explored by optimization algorithms.

Even if the proposed network was very simple, it is a first step in optimizing an embedded network by considering it as a whole system, and not as a simple combination of independent units. This is one of the main directions for future aircrafts optimization.

\section{REFERENCES}

[1] Schroeter, T.; Nya, B.H.; Schulz, D. "Potential analysis for the optimization of the electrical network of large modern civil and future single aisle aircraft and examples of the network capacity utilisation", ESARS 2010

[2] Roboam, X.; Sareni, B.; Andrade, A.D. "More Electricity in the Air: Toward Optimized Electrical Networks Embedded in More-Electrical Aircraft" IEEE Industrial Electronics Magazine

[3] Wen, Bo, Dushan Boroyevich, Paolo Mattavelli, Zhiyu Shen, and Rolando Burgos. "Influence of phase-locked loop on input admittance of three-phase voltage-source converters." In Applied Power Electronics Conference and Exposition (APEC), 2013 Twenty-Eighth Annual IEEE, pp. 897-904. IEEE, 2013.

[4] R.Pasterczyk, JM.Guichon, JL.Schanen, E.Atienza, "PWM Inverter output filter: cost-losses trade off and optimal design", IEEE trans on IAS- March/April 2009, Vol 45 n², pp.887-897

[5] B.Touré, JL.Schanen, L.Gerbaud, T.Meynard, R.Ruelland, J.Roudet, "EMC Modeling of Drives for Aircraft Applications: Modeling Process, EMI Filter Optimization and Technological choice" IEEE trans Power Electronics Vol 28 n³, February 2013, pp 1145 - 1156

[6] M. L. Nave, 3Power Line Filter Design for Switch-Mode Power Supplies", Van Nostrand Reinold, 1991, pp. 210

[7] M.Delhommais, G.Dadanema, Y.Avenas, F.Costa, JL.Schanen, C.Vollaire, "Design by Optimization of Power Electronics Converter Including EMC Constraints", IEEE-EMC Europe 2016 - Sep 5-9 2016, Wroklaw, Poland

[8] Tallam, R.M.; Skibinski, G.L.; Shudarek, T.A.; Lukaszewski, R.A., "Integrated differential-mode and common-mode filter to mitigate the effects of long motor leads on AC drives", ECCE 2010 Pages: 838 - 845

[9] Xuning Zhang; Dushan Boroyevich; Paolo Mattavelli; Jing Xue; Fei Wang, "EMI filter design and optimization for both AC and DC side in a DC-fed motor drive system" Applied Power Electronics Conference and Exposition (APEC), 2013 Pages: 597 - 603

[10] B. Revol, J. Roudet, J.L. Schanen, P.Loizelet, "EMI study of a three phase inverter-Fed Motor Drives", IEEE trans on IAS, Vol $47 \mathrm{n}^{\circ} 1$ January/Feb 2011, pp 223 - 231

[11] CADES software on http://www.vesta-system.fr/en/products/vestacades/

[12] Rautert, Tankred, and Ekkehard W. Sachs. "Computational design of optimal output feedback controllers." SIAM Journal on Optimization 7.3 (1997): 837-852.

[13] Burgos, Rolando, Dushan Boroyevich, Fred Wang, Kamiar Karimi, and Gerald Francis. "On the Ac stability of high power factor three-phase rectifiers." In Energy Conversion Congress and Exposition (ECCE), 2010 IEEE, pp. 2047-2054. IEEE, 2010. 